\title{
Analysis of the TMT mount control system
}

Peter M. Thompson, Douglas G. MacMynowski, Mark J. Sirota

Peter M. Thompson, Douglas G. MacMynowski, Mark J. Sirota, "Analysis of the TMT mount control system," Proc. SPIE 7012, Ground-based and Airborne Telescopes II, 70121P (10 July 2008); doi: 10.1117/12.790277

Event: SPIE Astronomical Telescopes + Instrumentation, 2008, Marseille, France 


\title{
Analysis of the TMT Mount Control System
}

\author{
Peter M. Thompson ${ }^{1 \mathrm{a}}$, Douglas G. MacMynowski ${ }^{\mathrm{b}}$, Mark J. Sirota ${ }^{\mathrm{b}}$ \\ ${ }^{a}$ Systems Technology, Inc., 13766 S. Hawthorne Blvd, Hawthorne, CA USA 90250 \\ ${ }^{\mathrm{b}}$ TMT Observatory Corporation, 2632 E. Washington Blvd., Pasadena, CA 91107
}

\begin{abstract}
The TMT mount control system provides telescope pointing and tracking. Requirements include wind disturbance rejection, offsetting time and accuracy, control system robustness, and the magnitude of response at structural resonances. A finite element model of the complete telescope has been developed and the transfer functions used for the control designs are presented. Wind disturbance, encoder, and wave-front-sensor models are presented that are used for the control design. A performance analysis translates the requirements to a required bandwidth. Achieving this bandwidth is important for reducing telescope image motion due to wind-buffeting. A mount control design is presented that meets the demanding requirements by maximizing low frequency gain and using structural filters to roll-off structural modes. The control system analysis includes an outer guide loop using a wave front sensor. Offsetting time and accuracy requirements are satisfied using feed-forward control architecture.
\end{abstract}

Keywords: extremely large telescopes, TMT, mount control, servo, azimuth, elevation, tracking, pointing, image jitter

\section{INTRODUCTION}

The Thirty Meter Telescope (TMT) is an azimuth/elevation (Az/El) telescope and when complete will be the world's largest optical/infrared telescope, see Figure 1. The primary mirror has 492 hexagonal segments, each segment is $1.44 \mathrm{~m}$ across the vertices.

The combined weight of the azimuth and elevation structure including the primary mirror (M1), the secondary mirror (M2), and the tertiary mirror (M3), instruments, and ancillary systems is 1670 tonnes. The Az structure is supported vertically on a $35 \mathrm{~m}$ diameter journal and six hydrostatic bearings pads, three pads on each side distributed equally over 70 degrees. The Az-axis is driven by a $35 \mathrm{~m}$ diameter linear motor. Lateral support is provided by a $3 \mathrm{~m}$ diameter pintle bearing and 5 hydrostatic pads. A tape encoder mounted at the location of the pintle bearing provides milli-arcsecond (mas) resolution.

The weight of the El structure including optics, instruments, and ancillary systems is 955 tonnes, The El structure is supported by two rockers, each with a radius of $10.75 \mathrm{~m}$ and supported vertically by two hydrostatic bearing pads, mounted twenty five degrees off vertical. Axial restraint is provided by two pairs of pads on each side of the axis. The El-axis is driven by a $10.75 \mathrm{~m}$ radius linear motor on each side. Tape encoders are mounted on each rocker, each with mas resolution. Further details about the telescope structural and mechanical designs are in a companion paper (Ref. 1).

Each axis is independently controlled

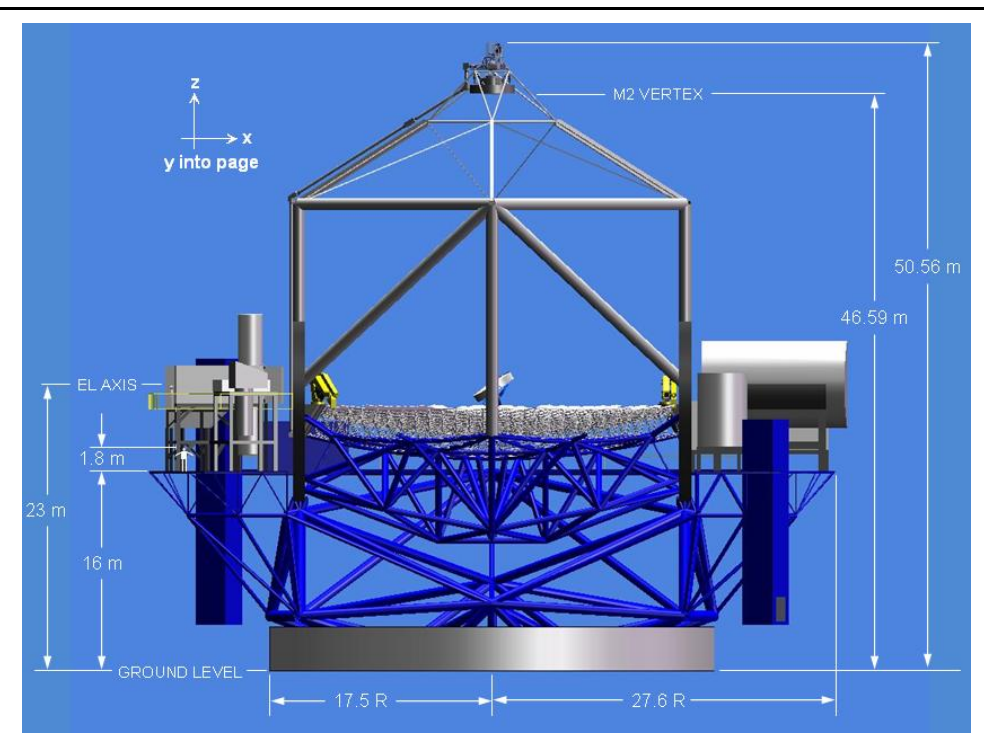

Figure 1: TMT Mechanical Design using nested position and guide loops. The motors utilize local analog feedback to control torque. Velocity

${ }^{1}$ pthompson@systemstech.com; phone (310) 679-2281; fax (310) 644-3887; www.systemtech.com 
measurements are inferred from the encoder position. The position loop controllers are digitally implemented with a sample rate of greater than $100 \mathrm{~Hz}$.

This paper presents the mount control architecture and compensators. After discussing requirements, frequency responses from the finite element model of the structure are presented, including low order approximations. A performance analysis using the lumped mass models is used to determine the position loop bandwidth needed to meet the wind rejection requirement. The mount control architecture is then presented, the design method described, followed by a listing of the compensators and the performance results. The analysis includes sensitivity of the results to wind disturbance model parameters, and finishes with autoguider and offsetting performance. Signals and systems are listed in Table 1. Parameters are defined in the text.

\begin{tabular}{|c|c|c|}
\hline \multicolumn{3}{|c|}{ Table 1: Signals and Systems } \\
\hline ElevRx, AzpRz, $R_{m}$ & $\mathrm{rad}$ & $\begin{array}{l}\text { Encoder angles: El, Az at pintle, } \\
\text { either axis }\end{array}$ \\
\hline $\mathrm{rcEl}, \mathrm{rcAz}, R_{l o s}$ & $\mathrm{rad}$ & Line of sight: El, Az, either axis \\
\hline wM2Fz, wM2Fx, $F_{w}$ & $\mathrm{~N}$ & Wind force on M2: El, Az, either axis \\
\hline ElevMx, AzMz, $T_{m}, T_{r}$ & $\mathrm{~N}-\mathrm{m}$ & $\begin{array}{l}\text { Motor torque: } \mathrm{El}, \mathrm{Az} \text {, either axis, } \\
\text { torque ripple }\end{array}$ \\
\hline$R_{\text {track }}, R_{\text {offset }}, R_{a}$ & $\mathrm{rad}$ & Command inputs \\
\hline$R_{\text {lag }}, R_{\text {low }}, R_{\text {high }}$ & $\operatorname{rad}$ & Filtered versions \\
\hline$e_{e n c}, e_{l u t}, e_{w f s}$ & $\mathrm{rad}$ & Measurement and look-up-table errors \\
\hline$g=R_{m} / T_{m}$ & $\mathrm{rad} / \mathrm{N}-\mathrm{m}$ & System used for position control \\
\hline$f_{\text {pid }}$ & $\mathrm{N}-\mathrm{m} / \mathrm{rad}$ & PID compensator \\
\hline$f_{s}, f_{n}, f_{a}$ & unitless & $\begin{array}{l}\text { Compensators: structural, notch, } \\
\text { autoguider }\end{array}$ \\
\hline$h_{e n c}, h_{w f s}$ & unitless & $\begin{array}{l}\text { Sensor models: encoder, } \\
\text { wave-front-sensor }\end{array}$ \\
\hline$l t f=g \times f_{n} \times f_{s}$ & unitless & Loop transfer function at $T_{m}$ \\
\hline
\end{tabular}

\begin{tabular}{|c|c|}
\hline Pointing & $\begin{array}{l}1 \text { arcsec RMS each } \\
\text { axis }\end{array}$ \\
\hline $\begin{array}{l}\text { Image jitter due to } \\
\text { wind }^{1}\end{array}$ & 5 mas RMS \\
\hline \multicolumn{2}{|c|}{ Tracking rates (arcsec/sec) } \\
\hline Azimuth & $\sim 0$ to $+/-885$ \\
\hline Elevation & 0 to $+/-15$ \\
\hline \multicolumn{2}{|l|}{ Slewing: Azimuth } \\
\hline Velocity & $2.4^{0} / \mathrm{sec}$ \\
\hline Acceleration & $0.2^{0} / \mathrm{sec}^{2}$ \\
\hline \multicolumn{2}{|l|}{ Slewing: Elevation } \\
\hline Velocity & $1.7^{0} / \mathrm{sec}$ \\
\hline Acceleration & $0.5^{0} / \mathrm{sec}^{2}$ \\
\hline \multicolumn{2}{|c|}{ Travel range: observing } \\
\hline Azimuth & $-270^{0}$ to $+270^{0}$ \\
\hline Elevation & $\begin{array}{l}1^{0} \text { to } 65^{0} \text { zenith angle } \\
\left(-1^{0} \text { to } 90^{0} \text { zenith }\right. \\
\text { angle; servicing) }\end{array}$ \\
\hline \multicolumn{2}{|c|}{$\begin{array}{l}\text { Offsetting (Nodding) times: } \\
\text { (Time to settle to within } 50 \text { mas) }\end{array}$} \\
\hline $1 \operatorname{arcsec}$ & $1 \mathrm{sec}$ \\
\hline $10 \operatorname{arcsec}$ & $2 \mathrm{sec}$ \\
\hline
\end{tabular}

\section{REQUIREMENTS}

The requirements on telescope performance are written in terms of absolute pointing and on-sky image jitter. How well the telescope meets these depends on the performance of the servo system, encoder system calibration, and the static telescope model. The paper deals only with servo performance: how well the telescope follows a command as measured by the encoder and the wave-front-sensor. The only disturbance considered, the dominant disturbance, is wind on the secondary mirror and its support structure.

Numerical requirements for the mount control system are listed in Table 2. The servo control has the most influence on image jitter and repositioning for short moves. Larger moves depend on the maximum velocity and acceleration. The maximum acceleration determines the motor torque requirements.

Requirements are set for each contribution to image jitter: the encoder (2 mas RMS each axis), the drive system ( 2 mas RMS each axis), the cable wrap (2 mas RMS each axis), and the two dimensional mean wind disturbance over wind speed and telescope orientation (5 mas RMS). For simplicity sake we assume that $100 \%$ of the wind force disturbs either the azimuth or elevation axis, the worst case scenario is when $100 \%$ of the wind disturbance is in elevation.

The image jitter requirements are ambitious; they not only push on the requirements for wind rejection by the enclosure and telescope structure design but also push the requirements for self generated disturbances. The TMT dome design (Ref. 3) significantly reduces the wind disturbance. 
The requirements in Table 2 are converted later in this paper to gain and frequency requirements for the mount control design. Additional requirements are added for control system robustness and for structural resonance magnitudes.

\section{CONTROLLED ELEMENT}

The controlled element for pointing and tracking control is the TMT elevation and azimuth structure. Inputs for the control designs are torque motors and wind force on the top end of the elevation structure including the M2 assembly. Outputs are angular position measured using encoders, and line of sight measured via wave front sensors. A finite element model of the structure has been developed and is used for the control design reported in this paper (Ref. 3, version dated 11-March-2008b).

\subsection{Frequency Responses}

The structural frequency response about the elevation and azimuth axis at 30 degrees zenith angle are shown in Figure 2 and 3 respectively. The damping ratio for all the modes is set to $0.5 \%$ in the finite element model. These are the collocated responses at the motor, the most important for mount control design. Although the azimuth encoder is located near the pintle bearing, some distance from the azimuth drives, the response looks very similar to the collocated response over most zenith angles. The locked rotor zero, the first big dip in the frequency response, is at about $3.7 \mathrm{~Hz}$ for both axes. The bandwidth for the mount control system will end up being about one third of the locked rotor frequency. The challenge is not to destabilize any of the numerous resonant modes above the locked rotor frequency.

The most important responses to determine image jitter due to wind are the M2 force to line-of-sight responses, Rlos/Fw (rcEL/wM2Fy and rcAZ/wM2Fx) respectively for El and Az-axes. Looking ahead, the locked rotor and closed loop versions of the wind responses are compared in Figure 8. The locked rotor response is important because it gives a lower bound on the closed loop performance

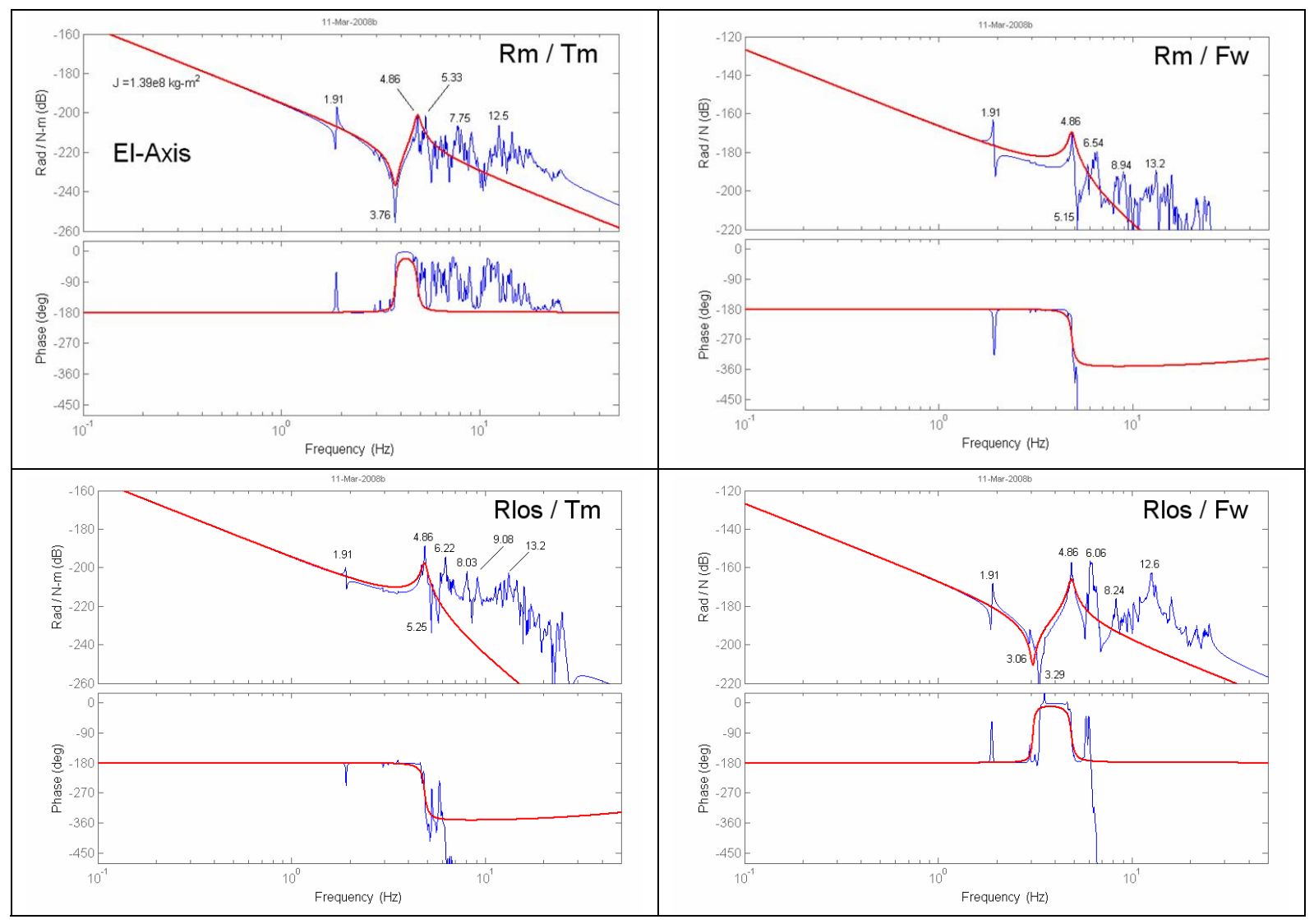

Figure 2: El-Axis Open Loop Frequency Responses 


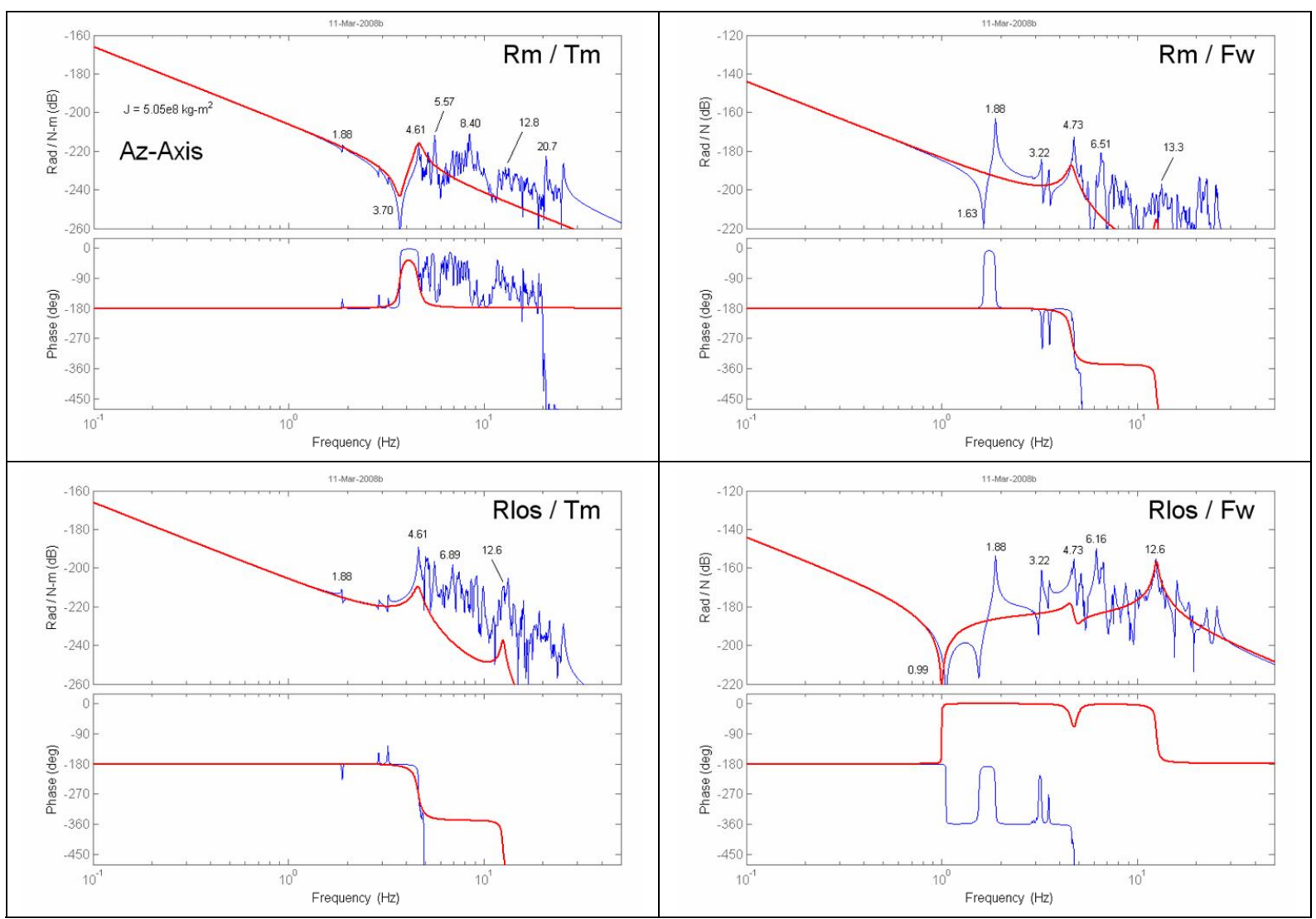

Figure 3: Az-Axis Open Loop Frequency Responses

\subsection{Lumped Mass Models}

The bold lines in Figure 2 and 3 are approximate, lumped mass models, illustrated in Figure 4. These models provide insight and are used for the performance analysis and time responses. Two masses separated by a spring and damper in parallel are used for the El-axis. A third mass is needed for the Az-axis to match the locked rotor response predicted using the finite element model. For the two-mass model define $J, f_{l r}$, and $f_{d}$ respectively as the total moment of inertia, the locked rotor zero and the differential resonance. Given these parameters it follows that $J_{1}=\left(f_{l r} / f_{d}\right)^{2} \mathrm{~J}, k_{1}=J_{2}\left(2 \pi f_{l r}\right)^{2}$, and $b_{1}$ is adjusted to fit the peak magnitude at $f_{d}$.

Transfer functions for the collocated motor response are listed below.

$$
\frac{\text { ElevRx }}{\text { ElevMx }}=\frac{1.2 \mathrm{e}-8[0.0225,3.76]}{(0)(0)[0.0291,4.86]}, \frac{\mathrm{AzpRz}}{\mathrm{AzMz}}=\frac{3.072 \mathrm{e}-9[0.0333,3.696][0.0187,12.54]}{(0)(0)[0.04143,4.605][0.01867,12.54]}
$$

The so-called "shorthand form" for displaying transfer functions is used above, which, besides being compact, provides quick reference to damping ratios and frequencies, and is defined by:

$$
a(b)[\zeta, f]=a(s+b /(2 \pi))\left[s^{2}+4 \pi \zeta f s+(2 \pi f)^{2}\right]
$$

The dominant poles and zeros have "generous" damping ratios in the 2 to 4 percent range even though the finite element model has only one-half percent damping for each individual mode. The (FEA) magnitude peaks are narrower than the lumped mass approximations and hence the FEA has the lower damping ratios. 


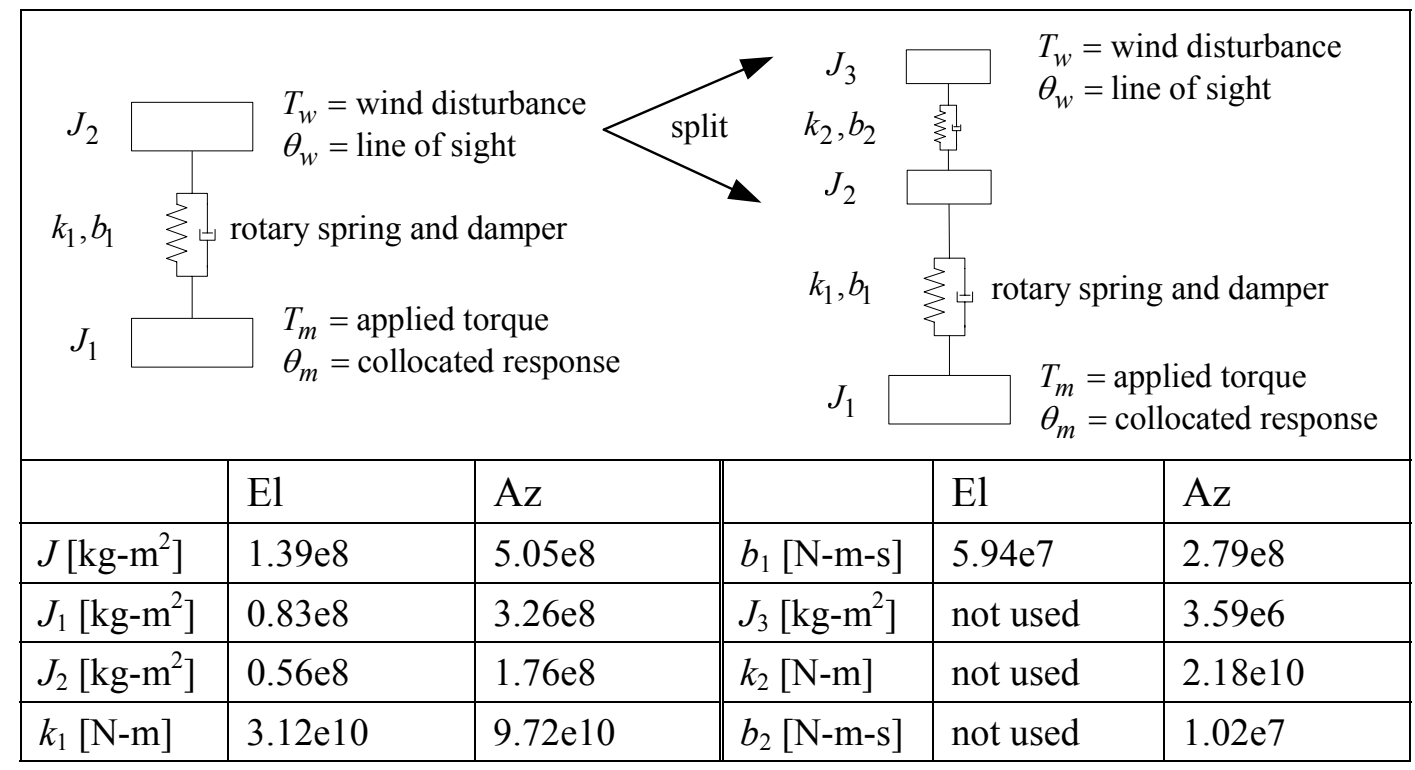

Figure 4: Lumped Mass Models.

\subsection{Wind Force Model}

Wind force on the M2 structure results in image jitter and is one of the significant limits to performance. Experimental data show that the wind force is well approximated using a von Karman spectrum:

$$
\Phi_{F_{w}}(f)=F_{S}^{2} \times \frac{0.77 / f_{0}}{\left[1+\left(f / f_{0}\right)^{2}\right]^{7 / 6}}(\mathrm{~N}-\mathrm{m})^{2} / \mathrm{Hz}
$$

where $F_{w}(t)$ is the wind time response, $F_{s}$ is the RMS wind force, and $f_{0}$ is the von Karman break frequency. The median far field wind speed is estimated to be $U_{\infty}=6.2 \mathrm{~m} / \mathrm{s}$. The wind speed at the M2 structure is $U_{M 2}=u_{2} \times U_{\infty}=0.93 \mathrm{~m} / \mathrm{s}$ using the reduction factor $u_{2}=0.15$ for the enclosure. The spectra parameters for the nominal wind model are:

$$
\begin{aligned}
& f_{0}=U_{M 2} / D=0.031[\mathrm{~Hz}] \\
& F_{S}=\frac{1}{2} \rho U_{M 2}^{2} C_{d} A=7.35[\mathrm{~N}]
\end{aligned}
$$

where $D=30 \mathrm{~m}$ is the diameter of the dome aperture, $\rho=0.82 \mathrm{~kg} / \mathrm{m}^{3}$ is the air density at $4000 \mathrm{~m}, C_{d}=1.5$ is the estimated drag coefficient, and $A=13.8 \mathrm{~m}^{2}$ is the area of the $\mathrm{M} 2$ structure and support. Note that the $f_{0}$ break frequency varies linearly with wind speed and the $F_{s}$ RMS wind force varies with wind speed squared. In this report the wind model is approximated as unit intensity white noise through a first order filter:

$$
\Phi_{F_{w}}(s)=f_{F_{w}}(s) f_{F_{w}}(-s), \text { where } f_{F_{w}}(s)=\frac{\alpha F_{s}}{s+\beta}, \begin{aligned}
& \alpha=\sqrt{2 \beta} \\
& \beta=2 \pi \times 0.900 \times f_{0}
\end{aligned}
$$

The break frequency at $f_{0} \mathrm{~Hz}$ in the linear model is decreased slightly to account for the slower roll-off of the first order filter. The wind force is applied at a point near the M2 structure a distance $r_{M 2}$ from the axis of rotation, so that the torque due to the wind is $T_{w}=F_{w} \times r_{M 2}$.

It is seen that most of the wind energy occurs below about $0.1 \mathrm{~Hz}$; nevertheless the control system bandwidth must be much more than this in order to achieve high gain in the low frequency range. A more comprehensive wind model is used for formal compliance checking (Ref. 3). The wind force is not treated as a point source but is distributed across the telescope top end (M2, laser-launch telescope) and its support structure. The spatial decorrelation of turbulence along the support structure members results in roll-off faster than the von Karman structure and hence a higher order spectral model is used. The more complicated wind model also includes wind across the M1 mirror, which depends on the enclosure vent openings. Trade studies with different dome radii and different types of openings have been conducted with the objective of reducing the wind force on the telescope top end. The wind force is reduced by (1) using a circular 
opening (hence minimum aperture area), (2) choosing the dome size and using flaps around the opening so that the telescope top end structure is below the shear layer that forms over the aperture, (3) minimizing the cross-sectional area of the telescope top end.

\subsection{Encoder Sensor Model}

The encoder sensor model is a delay to account for processing, and the sensor noise is additive quantization noise $\left(q_{\text {enc }}\right.$ is one least significant bit of the encoder, $R$ is the actual position at the encoder).

$$
\begin{gathered}
R_{\text {enc }}=h_{\text {enc }}(s) R+n_{\text {enc }}, \text { where } \\
h_{\text {enc }}(s)=e^{-s \tau_{e n c}} \approx \frac{1}{\tau_{\text {enc }} s+1}, n_{\text {enc }}=\text { Gaussian white noise with intensity } q_{\text {enc }}^{2} / 12
\end{gathered}
$$

The processing delay is less than one msec, and here zero is used. The intensity is the variance of a uniform random variable with assumed width $q_{\text {enc }}=2$ mas. At the speed $100 \mathrm{mas} / \mathrm{sec}$ and with a sample rate of $100 \mathrm{~Hz}$, an encoder mark is passed every two samples. A digital model with a sample and hold device is a more accurate model at this speed and below.

\subsection{Wave Front Sensor Model}

A wave front sensor model is shown in Figure 5. The continuous input signal (photons) are averaged over $\tau_{i}$ seconds, followed by a transmission delay of $\tau_{p}$ seconds, then a sample and hold, with a new signal every $\tau_{w}$ seconds. The wave front signal is buffered and sampled every $T$ seconds by the mount position controller. The axis transformations and additive errors are not included in the sensor model used here.

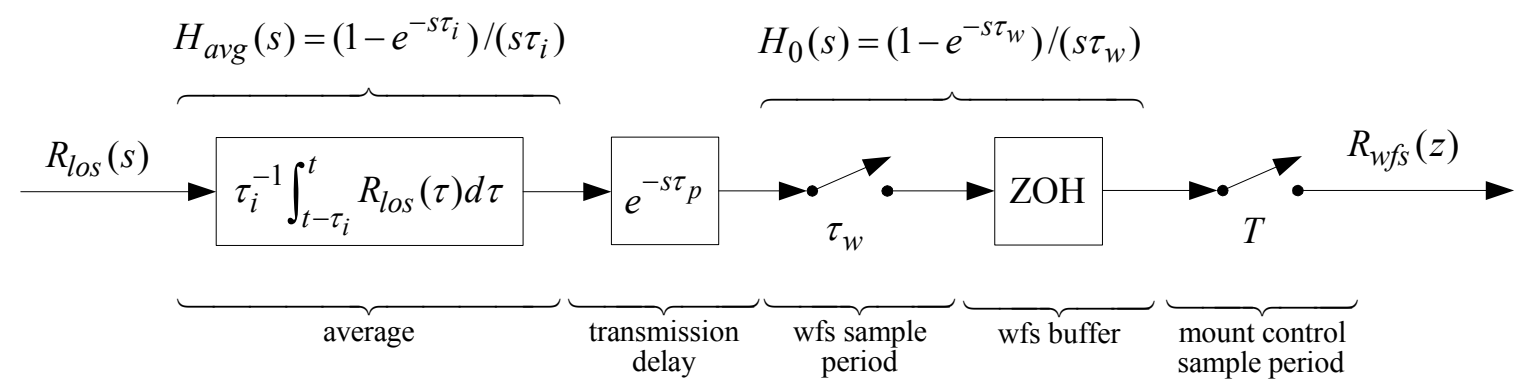

Figure 5: Wave Front Sensor Model

Impulse response models of the integration and the sample-and-hold are listed over these blocks and have the same form. A sensor model from the LOS input to the signal used by the position controller is:

$$
\operatorname{LOS}_{w f s}(z)=\left[H_{0}^{t_{w}}(s) e^{-s t_{p}} H_{\text {avg }}^{t_{i}} L O S\right]^{T}
$$

The superscript $T$ means "take the z-transform of the impulse response." This is a linear time varying operator, since the shifted input and output are not the same, but the effects of the time variation are small, and an approximate, Laplace transform sensor model is:

$$
H_{w f s}(s)=H_{0}^{t_{w}}(s) e^{-s t_{p}} H_{a v g}^{t_{i}}(s) \approx \frac{1}{s t_{w} / 2+1} \times \frac{-s t_{p} / 2+1}{s t_{p} / 2+1} \times \frac{1}{s t_{i} / 2+1} \approx \frac{1}{s \tau_{w f s}+1}
$$

The effective delays are added together in the simpler, first order model, where $\tau_{w f s}=\tau_{w} / 2+\tau_{p}+\tau_{i} / 2$, and the first order version is the sensor model used here. Estimates of wave front sensor performance are 5 to 10 samples per second. Use the $10 \mathrm{~Hz}$ estimate, with $\tau_{w}=\tau_{i}=100 \mathrm{msec}, \tau_{p}=0$, and hence $\tau_{w f s}=100 \mathrm{msec}$. The simple sensor model has a single pole at $10 \mathrm{rad} / \mathrm{sec}$. The more detailed models are needed if the integration time becomes large, on the order of one second or more. 


\section{PERFORMANCE ANALYSIS}

A performance analysis using simplified models is conducted to determine the bandwidth needed to meet the wind jitter requirement. Bandwidth is defined as the unit magnitude crossover frequency of the loop transfer function, and it will turn out that the bandwidth should be just over one Hertz.

Start the analysis by considering the wind jitter of the locked rotor response, which is relevant because the structure with the motors locked in place must be stiff enough to meet the wind jitter requirement. Using the nominal wind model it follows that $\sigma_{L O S}=1.37$ and 1.31 [mas RMS] respectively for the El and Az-axes. The RMS values are below the requirement of 5 mas and so it is concluded that the structure, not including the drives, by itself is stiff enough to meet the wind rejection requirement.

The wind induced RMS response of the closed loop El-axis is approximated by:

$$
\sigma_{L O S} \approx 0.79 F_{S} / f_{c}^{2.2}=5.0[\text { mas RMS }] \text { for } F_{S}=7.35[\mathrm{~N}], f_{c}=1.07[\mathrm{~Hz}]
$$

This approximation will shortly be derived using a curve fit. For now it is noted that the scale factor 0.79 depends on the moment of inertia, the robustness margins and the von Karman break frequency. Using this approximation it is seen that a crossover frequency of just over $1 \mathrm{~Hz}$ meets the wind RMS requirement, and it is seen that the dependence on the crossover frequency is slightly more than inverse squared, and so a $10 \%$ increase in the crossover frequency results in slightly more than a $20 \%$ decrease in the wind jitter. There is a big payoff in the El-axis for even small increases in the crossover frequency. Conversely small decreases in bandwidth can result in a large increase in image jitter. The Az-axis has about 4 times the moment of inertia, and it will turn out about one fourth the RMS tracking error. Tracking requirements are easily met in the Az-axis.

The details of the performance analysis are now presented. A proportional-integral-derivative (PID) controller is designed and the closed loop RMS wind jitter computed for a range of parameters.

\subsection{PID Design}

The feedback architecture is shown in the top right of Figure 6. The three gains of the PID controller are computed as functions of three design parameters, which are exactly achieved for a rigid body system, and close enough otherwise. Define the three design parameters:

$$
\begin{aligned}
& f_{c}[\mathrm{~Hz}]=\text { unit magnitude crossover frequency } \\
& \text { PM }[\mathrm{deg}]=\text { phase margin } \\
& \text { LGM }[\mathrm{dB}]=\text { lower gain margin }
\end{aligned}
$$

The phase margin is extra phase lag that destabilizes the system, and the lower gain margin is the gain reduction that destabilizes the system. It follows non-obviously that:

$$
\begin{aligned}
& k_{i}=-\beta \omega_{c}^{2} /\left(\operatorname{lgm} \times \operatorname{sqrt}\left(1+\beta^{2}\right)\right) \quad \beta=\tan (90+\mathrm{PM}) \\
& k_{p}=\beta\left(k_{i}-\omega_{c}^{2}\right) / \omega_{c} \quad \text { where } \operatorname{lgm}=10^{\mathrm{LGM} / 20} \\
& k_{r}=\operatorname{lgm} \times J \times k_{i} / k_{p} \quad \omega_{c}=2 \pi f_{c}
\end{aligned}
$$

The rate gain varies with the moment of inertia $J$ and hence is a very large number. The other gains in the structure defined here end up being modest sized numbers. The derivation is not given but the first step is that $k_{i}=\omega_{\operatorname{lgm}}^{2}$, where $\omega_{\operatorname{lgm}}$ is the frequency where the first -180 degree crossover occurs.

\subsection{Required Unit Magnitude Crossover - Elevation Axis}

Use the moment of inertia for the elevation axis. The design parameters and resulting controller gains are:

$$
\begin{gathered}
J=1.39 \mathrm{e} 8\left[\mathrm{~kg}-\mathrm{m}^{2}\right], f_{c}=1[\mathrm{~Hz}], \mathrm{PM}=60[\mathrm{deg}], \mathrm{LGM}=12 \mathrm{~dB}, \mathrm{DM}=\mathrm{PM} /\left(360 f_{c}\right)=167[\mathrm{msec}] \\
k_{r}=6.22 J=8.66 \mathrm{e} 8[\mathrm{~N}-\mathrm{m}-\mathrm{sec}], k_{p}=3.17[1 / \mathrm{sec}], k_{i}=4.96\left[1 / \mathrm{sec}^{2}\right]
\end{gathered}
$$




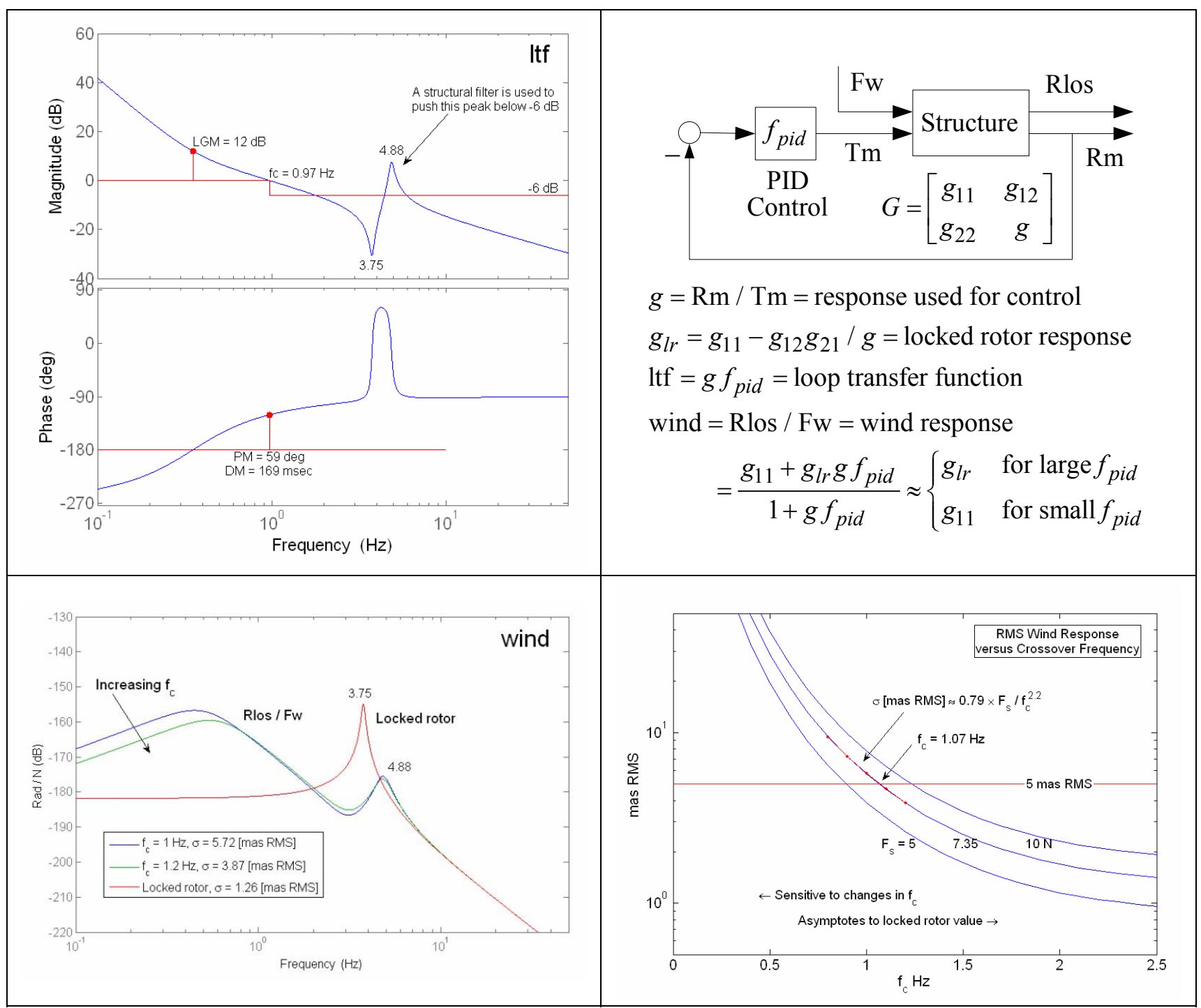

Figure 6: Elevation axis performance analysis

Loop transfer function: The ltf is plotted on the left side of Figure 6 using the lumped mass model. The crossover and phase margin are shifted slightly due to the resonance. The differential resonance at $4.88 \mathrm{~Hz}$ is stable, and in the next design a structural filter is used to bring the peak magnitude below the $-6 \mathrm{~dB}$ line. The generous phase margin of 60 degrees leaves room for the structural filter phase lag.

Delay margin: The delay margin (DM) is the extra delay that destabilizes the control loop. The delay margin is better than phase margin as a way to account for the "effective delay" that will occur due to the encoder, motor, and power amplifier. (Better because a phase margin is always tied to a particular crossover frequency, whereas the delay margin is not). All lags look like delay at frequencies well below their break frequency, called an "effective delay." The sensors and actuators are expected to have an effective delay less than $\tau=5 \mathrm{msec}$, which is much less than the allowance of 167 $\mathrm{msec}$ in the PID design, and hence the design is robust. The effective delay of $5 \mathrm{msec}$ translates to 1.8 degrees of phase margin at the $1 \mathrm{~Hz}$ crossover frequency, equivalent to a first order lag with a corner frequency of $1 /(2 \pi \tau)=32 \mathrm{~Hz}$.

Bandwidth: In this paper "bandwidth" is defined as the unit magnitude crossover frequency $f_{c}$ of the loop transfer function. Bandwidth can alternatively be defined based on closed loop responses, such as the $-3 \mathrm{~dB}$ crossover of the sensitivity function $S=1 /(1+l t f)$, or the $-3 \mathrm{~dB}$ crossover of the complementary sensitivity $T=l t f /(1+l t f)$. All three of 
these definitions give the same bandwidth for a $k / s$ system, but they differ in general. Neither of the closed loop versions applies to the wind rejection problem, which is why the unit magnitude crossover is used.

Wind response: Now please turn your attention to the lower left of Figure 6, which compares the locked rotor and closed loop wind responses. The closed loop version is plotted for two different crossover frequencies. The "hump" at about one-half $\mathrm{Hz}$ is gradually pushed down to the locked rotor line as the crossover frequency increases. The achieved RMS wind responses are included in the figure. A crossover of $1 \mathrm{~Hz}$ does not quite meet the 5 mas requirement, whereas a crossover at $1.2 \mathrm{~Hz}$ is comfortably below the requirement.

Required crossover: In the bottom right part of Figure 6 the crossover frequency is varied over a range, everything else is held constant, and the resulting RMS wind response is plotted. The RMS value depends linearly on the input wind force, and the response line is repeated for one lower and one higher value of wind force. It is from this plot that it is seen that a crossover frequency of $1.07 \mathrm{~Hz}$ meets the 5 mas requirement.

RMS Curve Fit: An analytical expression for the RMS value $\sigma_{l o s}$ was not found, and in any case would be complicated. As an alternative several points above and below the 5 mas requirement are used for the nonlinear curve fit $\sigma_{L O S}=\alpha F_{s} /$ $f_{c}^{n}$, where it is found $\alpha=1.00$ and $n=2.2$. Lowering the PM and lowering the LGM will decrease the coefficient, for example 45 degree PM decreases the coefficient to $\alpha=0.78$.

\section{MOUNT CONTROL DESIGN}

The feedback and feed-forward architecture is shown in Figure 7. The parts are discussed, followed by the control designs for each axis and surveys of the results.

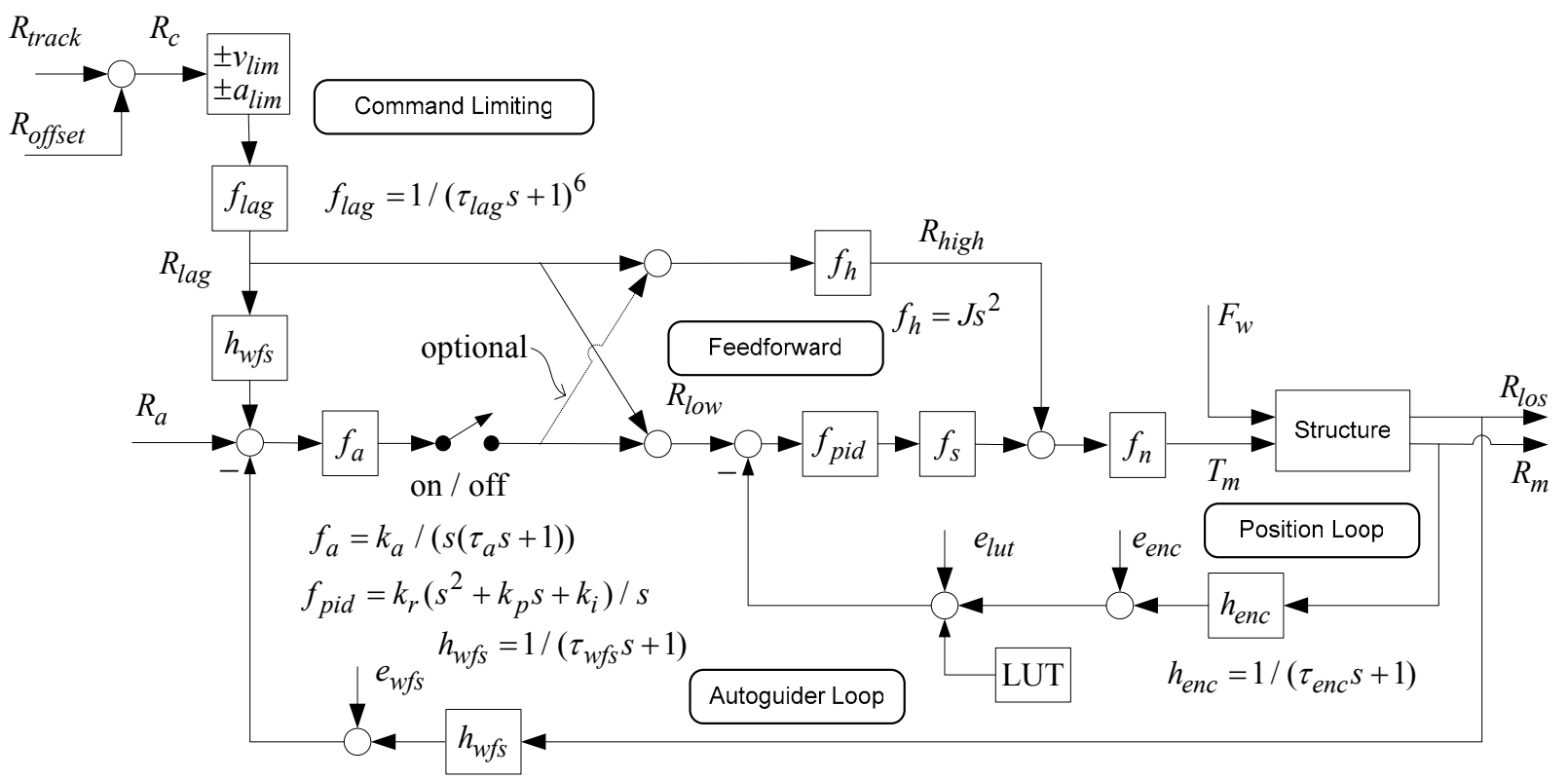

Figure 7: Mount Control Architecture

\subsection{Architecture}

Exogenous inputs: The wind force $F_{w}$ and torque ripple $T_{r}$ are disturbance inputs with approximately the same disturbance rejection properties. The position command $R_{c}=R_{\text {track }}+R_{\text {offset }}$ is the track to be followed plus an offset for nodding and/or an autoguider update. The input $R_{a}$ is an option for the autoguider. Sensor error signals for the encoder and wave front sensor are respectively $e_{e n c}$ and $e_{w f s}$. The look-up-table (LUT) input is a correction added to the encoder output and has its own error signal $e_{l u t}$.

Position loop: The inner loop uses position feedback from the encoder. Rate feedback is not used; rate is internally generated in the PID controller. The PID controller is augmented with a structural filter $f_{s}$ and a notch filter $f_{n}$, which reduce the peak magnitudes of the structural resonances, and serve double-duty by reducing encoder noise. The notch 
filter is separated so that the feedforward signal is also notched. Gains will be moved around in the detailed implementation - most of the needed large gain will occur in the power amplifiers.

Autoguider loop: The autoguider loop uses a line-of-sight measurement obtained from a wave-front-sensor. The compensator $f_{a}$ is an integrator times a first order lag. The autoguider loop can be turned on or off. When off the position loop is used for slewing maneuvers. Autoguider input can either be at $R_{a}$ or $R_{o f f s e t}$. The latter provides protection against torque saturation. The optional path after $f_{a}$ provides feedforward to the motor torque, which has the effect of increasing the bandwidth of the autoguider response. Axis transformations are not included in this analysis.

Command limiting: The position command $R_{c}$ is input to a nonlinear circuit that limits the acceleration and velocity, followed by the lag filter $f_{\text {lag }}$ that limits the jerk, resulting in $R_{\text {lag }}$. The acceleration limit prevents motor torque saturation.

Feedforward: The signal $R_{\text {lag }}$ is injected into the feedback loop in three different places: 1) a filtered version into the autoguider loop, the filter prevents overshoot that would otherwise occur due to the wave front sensor lags, 2) an unfiltered version into the position loop input, and 3) the scaled second derivative adds to the torque command. The block $f_{h}=J \times s^{2}$ inverts the low-frequency part of the controlled element. The acceleration is not computed directly but is an output of $f_{\text {lag }}$ and (optionally) $f_{a}$. To explain the feedforward network: if $h_{e n c}=f_{n}=1$ and $R_{m}=R_{l o s}=\left(1 /\left(J \times s^{2}\right)\right) T_{m}$ then $R_{m} / R_{\text {lag }}=1$. Qualitatively, the feedforward network injects signals that exactly zero out the feedback errors.

\subsection{Control Problem}

The control problem has the following objectives:

Maximize the low frequency gain below crossover, which will maximize wind rejection. To do this, maximize $f_{c}$, the unit magnitude crossover frequency of the ltf measured at $T_{m}$, while at the same time just meeting the robustness requirements. The crossover frequency shall not be less than $1 \mathrm{~Hz}$.

The robustness requirements are at least 35 degrees phase margin and $6 \mathrm{~dB}$ plus and minus gain margin. The phase margin requirement takes into account an expected loss of 5 degrees phase margin due to sample delay, sensor, motor, and power amplifier lags. This is equivalent to an effective delay for these subsystems of about $15 \mathrm{msec}$. Of course, additional gain or phase margin is beneficial.

Design a structural filter so that all of the resonances above $4 \mathrm{~Hz}$ have peak magnitudes less than $-6 \mathrm{~dB}$. Gain reduction comes with phase lag, and the challenge is to roll-off quickly while maintaining adequate robustness margins.

Design the autoguider to maximize the unit magnitude crossover of the ltf measured at $R_{l o s}$, without increasing the RMS wind jitter, and maintaining a first-order-like step response. The crossover frequency shall not be less than $0.1 \mathrm{~Hz}$.

A classical design approach is used, with a PID compensator as described earlier, and with a fixed form structural filter. The PID and structural filter parameter are adjusted in an ad-hoc manner. Formal optimization was not attempted. The structural filter is split into two parts, so that the feedforward signal goes through the additional notch filters, and has the following form:

$$
f_{s}=\underbrace{\frac{f_{z}^{2}}{f_{p}^{2}} \times \frac{\left[0.1, f_{p}\right]}{\left[0.1, f_{z}\right]}}_{\text {Notch with gain reduction }} \times \underbrace{\frac{\sqrt{c / b}(b)}{(c)}}_{\text {Lead-lag }} \times \underbrace{\frac{2.5 \times 20 \times 8 \times \pi^{3}}{(2.5)[0.5,20]}}_{\text {Roll-off }}, f_{n}=\underbrace{\frac{\left[0.04, f_{n 1}\right]}{\left[0.2, f_{n 1}\right]} \times \frac{\left[0.04, f_{n 2}\right]}{\left[0.1, f_{n 2}\right]}}_{\text {Additional notches }}
$$

The notch with $f_{z}<f_{p}$ provides rapid gain reduction with minimal phase loss. The notches centered at specific frequencies keeps resonant peak magnitudes at these frequencies below $-6 \mathrm{~dB}$. The lead-lag boosts the phase lead at crossover, countering the phase loss of the structural filter. The PM parameter in the PID design does the same thing. The roll-off lowers all of the resonant peaks, and in additional reduces the response to sensor noise. The notch with gain reduction and with additional roll-off is essentially the same as an elliptic filter, but direct adjustment of the filter parameters was found to be a better alternative than adjusting the elliptic filter parameters. Similarly, direct adjustment of PID and filter parameters is considered to be a better alternative than adjusting terms in a LQG cost function.

The structural filter is "highly tuned" to the finite element structural model, and needs to be adjusted as the finite element model changes and again when the actual structural response is identified. The structural resonances change with zenith angle, enough so that some of the compensator parameters need to be scheduled with zenith angle. This has been done, 
but only the thirty degree zenith case is reported here. Backing off on the crossover frequency $f_{c}$ eliminates the need for the complicated structural filter, and scheduling and tuning, but at the expense of increased wind response.

\subsection{El-Axis Control Survey}

A graphical survey of the El-axis mount control is on the left side of Figure 8. Included are the Bode plots of the $l t f$ at $T_{m}$ and the wind response. From the ltf Bode it is seen the resonances at 4.85 and $5.37 \mathrm{~Hz}$ limit the achievable performance. The $l t f$ "cuts through" the resonance at $1.90 \mathrm{~Hz}$, actively damping this mode. This is not a stability problem because the phase at this frequency rises, but it does result in a unit magnitude crossover at $1.95 \mathrm{~Hz}$ with a lower phase margin and delay margin. On the wind rejection Bode plot it is seen the 5 mas image jitter requirement is met when using the nominal wind model, with or without the autoguider loop closed. The compensators and numerical results for both axes are listed in Table 1

\subsection{Az-Axis Control Survey}

A graphical survey of the Az-axis mount control is on the right side of Figure 8. The unit magnitude crossover is limited by the resonance that peaks up at $5.57 \mathrm{~Hz}$. The image jitter requirement is easily satisfied for the Az-axis, due mainly to the increased moment of inertia, and so there is room to back off on the unit magnitude crossover.

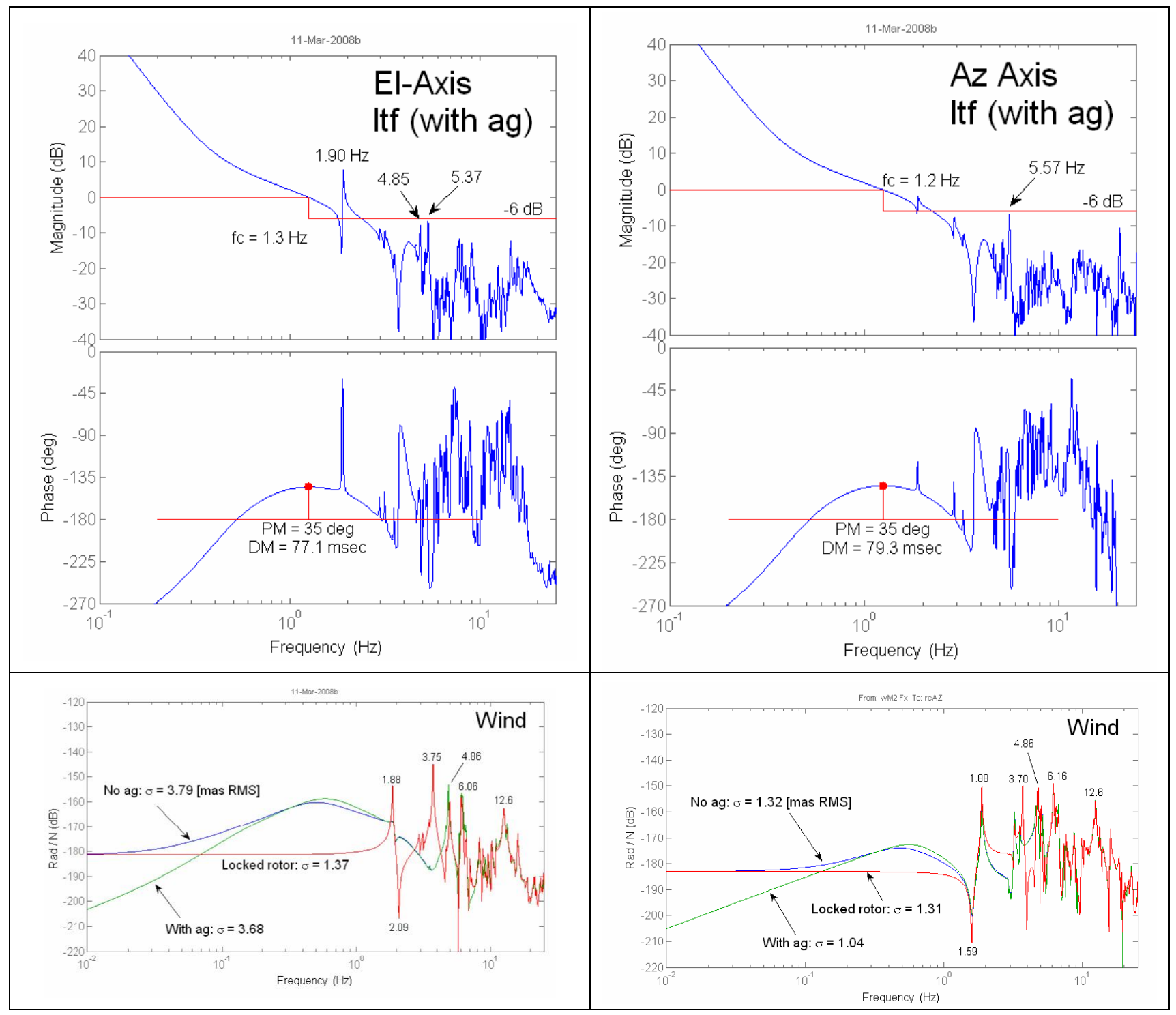

Figure 8: Mount Control Design Surveys (Zenith 30 deg) 
Table 1: Mount Control Summary

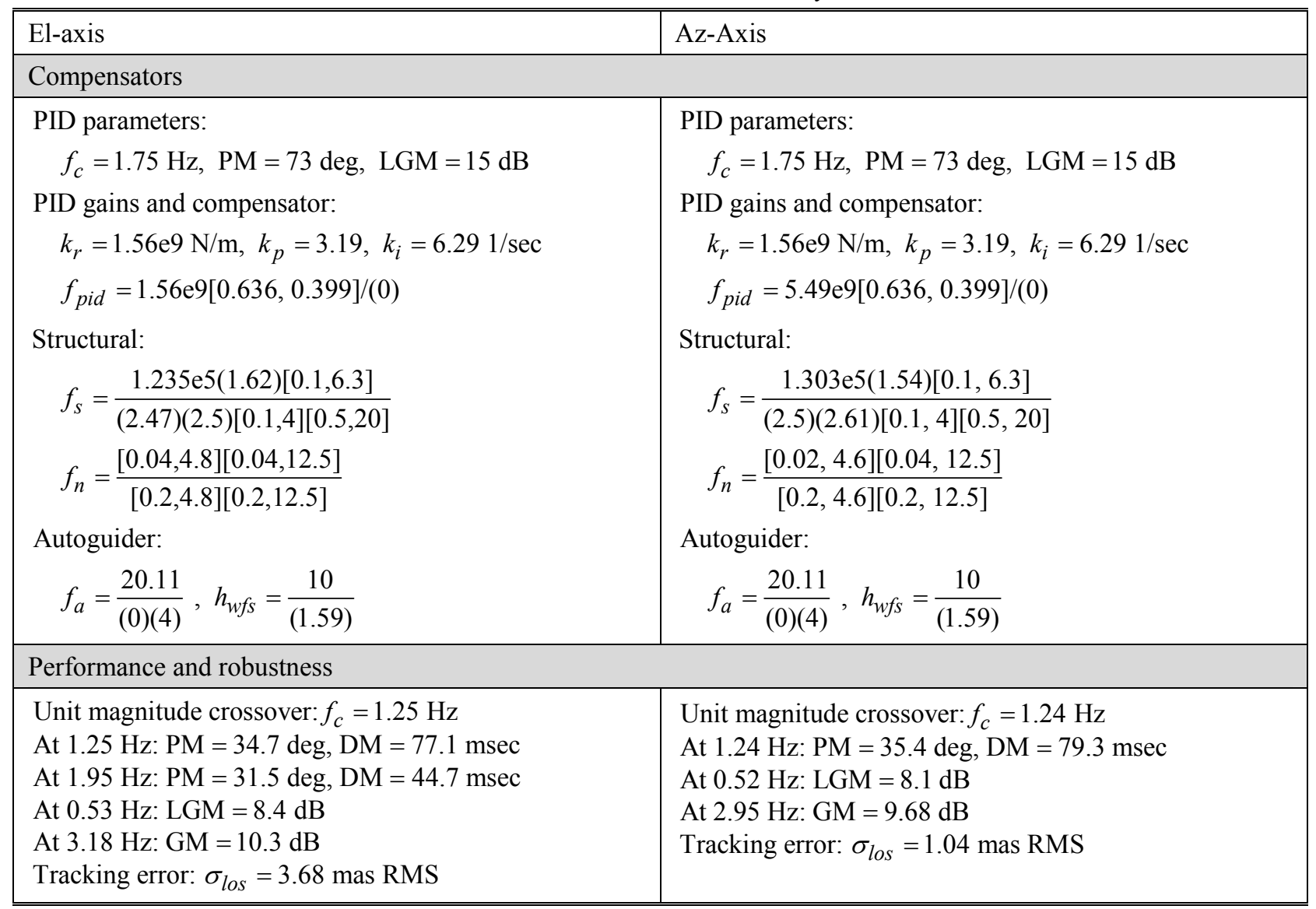

\subsection{Autoguider}

It is counter-intuitive that the high sample rate wave-front-sensors that are now available do not necessarily reduce the wind-induced tracking error. The extra integration in the autoguider loop has extra phase lag, which must be compensated with extra lead, resulting in about the same $f_{c}$, and hence the same wind response. There is still freedom to tradeoff gain in the autoguider and position loops, using the optional feedforward path in Figure 7. The El-axis autoguider is surveyed in Figure 9, showing the LTF at the autoguider error and the closed loop unit step response from the $R_{a}$ input. The two lines in each plot are for the conventional implementation and with the feedforward option. The latter has the higher autoguider and lower position loop bandwidth, and would seem to be the obvious choice, but comes at the price of increased tracking error (3.66 increases to 4.69 mas RMS using the nominal wind model) and increased chance of motor torque limiting. It is possible that a better optimized set of gains will recover the lost tracking error. The unit magnitude crossover frequency in Figure 9 is labeled $f_{c a}$ and marked with dots. The structural resonance at $6.22 \mathrm{~Hz}$ is what limits the autoguider crossover, and results in the slight oscillations seen in the faster step response. These oscillations disappear if $R_{\text {offset }}$ is used as the autoguider command input.

\subsection{Allowable Wind Models}

A wind model is actually a set of wind models with an accompanying probability density function, this is the approach used in Ref. 3 for compliance checking. The approach in this report is to work backwards from the structure and controller and determine the set of wind models for each axis that meet the wind RMS requirements. The results are shown in the Figure 10 parameter planes. The shaded region is the combination of wind force $F_{s}$ and von Karman break frequency $f_{0}$ that meets or exceeds the wind guide error on the boundary. The boundary lines change based on the moment of inertia and the control compensators. The square root line that slashes across the plot shows the dependence of the wind model parameters on the far field external wind speed. Where the boundary line becomes vertical there is very little sensitivity to changes in the von Karman break frequency. The first order models can be used to upper bound 
higher order and more comprehensive wind models. The "design point" of $F_{s}=7.35 \mathrm{~N}$. and $f_{0}=0.031 \mathrm{~Hz}$. is inside the shaded region for both axes.

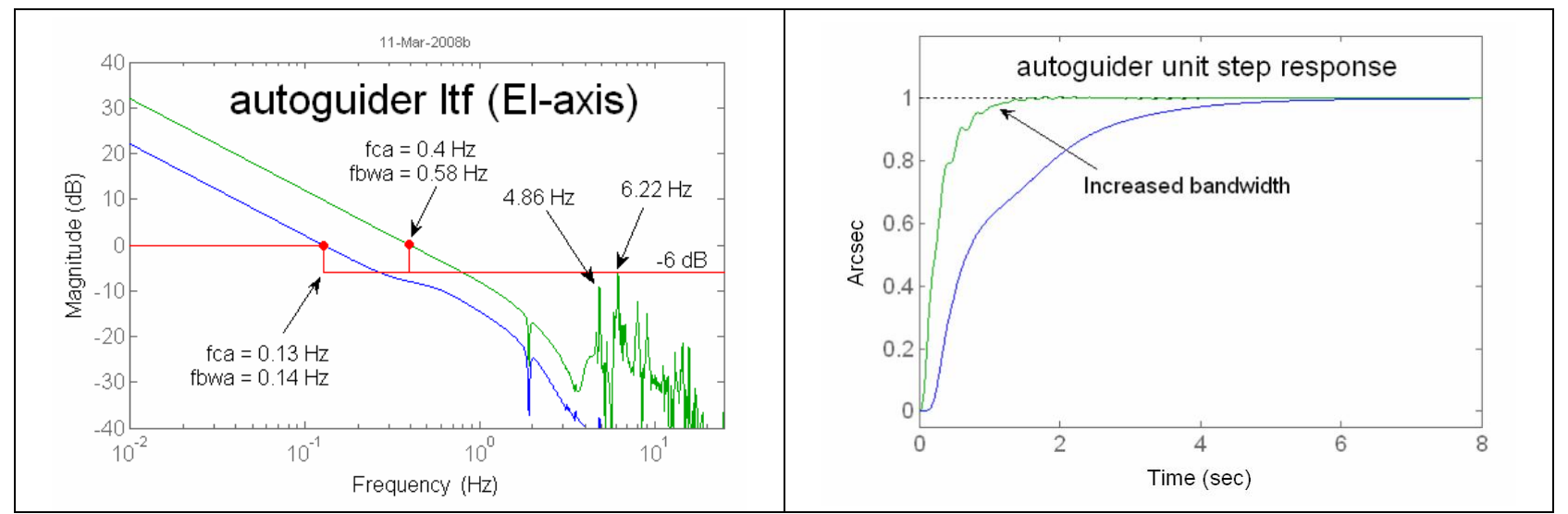

Figure 9: Autoguider Survey (Zenith 30 deg)

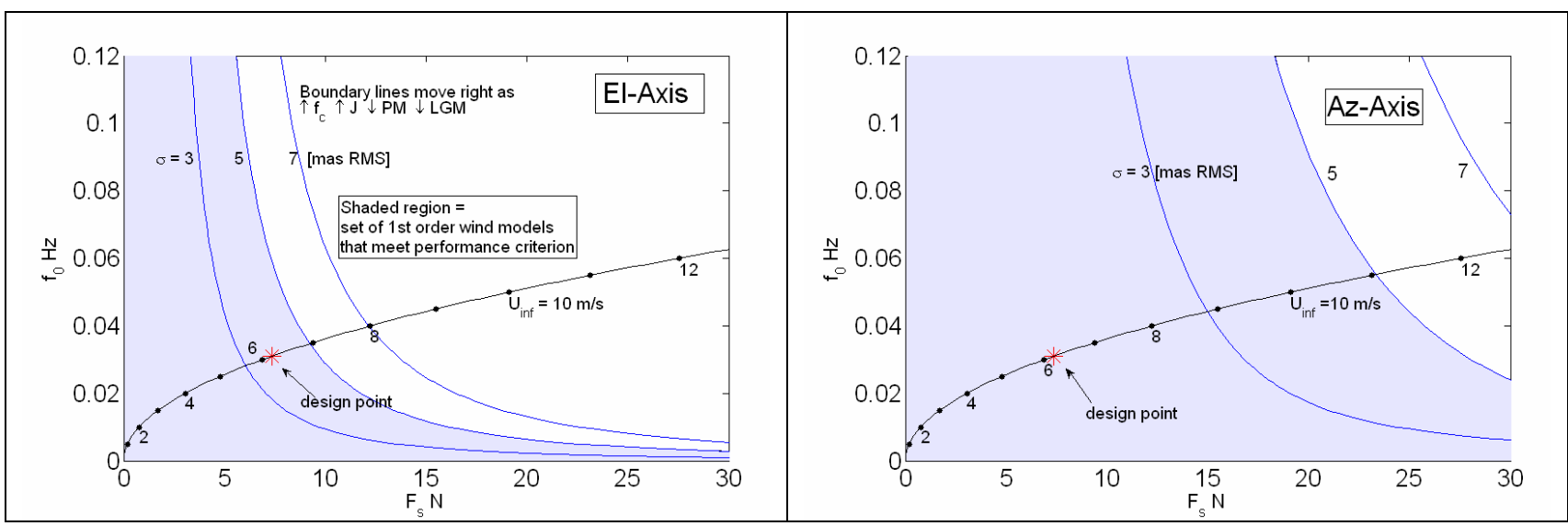

Figure 10: Mount Control Design Surveys (Zenith $30 \mathrm{deg}$ )

\subsection{Offset Step Responses}

Different size offsets are injected into the $R_{\text {offset }}$ input in Figure 7 and the resulting responses are compared in Figure 11. The velocity and acceleration limiter block in Figure 7 converts the offsets into minimum time trajectories. The one and two arcsec offsets settle to within 0.02 arcsec respectively within 1 and 2 seconds. Larger offsets have a maximum overshoot of 2 arcsec.

Minimum time responses are reviewed, where $V_{\max }$ is the maximum velocity, $A_{\max }$ is the maximum acceleration, and $J$ is the moment of inertia. The maximum torque is $T_{\max }=J \times A_{\max }$. The time to reach maximum velocity is $t_{v \max }=V_{\max } / A_{\max }$, during which the axis moves $R_{v \max }=V_{\max }^{2} /\left(2 A_{\max }\right)$. For offsets less than $2 R_{v \max }$ the minimum time is $t_{\min }=2 \operatorname{sqrt}\left(R_{\text {offset }}\right.$ $\left(A_{\max }\right)$, during which the maximum velocity reached is $V_{\max }=\operatorname{sqrt}\left(R_{\text {offset }} \times A_{\max }\right)$. For larger offsets $t_{\min }=2 t_{\text {vmax }}+\left(R_{\text {offset }}-\right.$ $\left.2 R_{v \max }\right) / V_{\max }$. The $f_{\text {lag }}$ filter after the limiter adds about 1.0 second to any size offset, but has the desirable effects of limiting overshoot and preventing spikes in the torque response.

\section{CONCLUSIONS}

The TMT mount control system in this model-based design has met the performance requirements for image jitter due to wind disturbance and for offset times and accuracy. Good robustness margins are satisfied. The results of this study have been used to set hardware requirements for subsystems such as structural resonance locations, enclosure wind attenuation, sensor accuracy, location and bandwidths; and motor torques. 
The image jitter performance requirement is challenging and meeting these requirements depends on the assumptions built into the stochastic wind model. Other sources of image jitter error, such as cable wrap friction, torque ripple, and hydrostatic bearing stiffness and friction are not reported here but the same models and tools have been used for these types of trade studies.

The image jitter error margin for the Az-axis is a generous 4:1, helped mainly by the large moment of inertia, in this respect size helps. The same error margin for the El-axis is about $36 \%$, with the main limitation being the locked rotor structural resonance at $3.7 \mathrm{~Hz}$. Performance is maximized by using a structural filter that is tuned based on the locked rotor and other structural resonances. Re-tuning will be required as the model changes and the actual structure is identified.

The mount control system is now at the preliminary design phase and is ready for progressing through the detailed design and implementation phases. Lessons learned during the Keck telescope development (Ref. 2) will be applied.

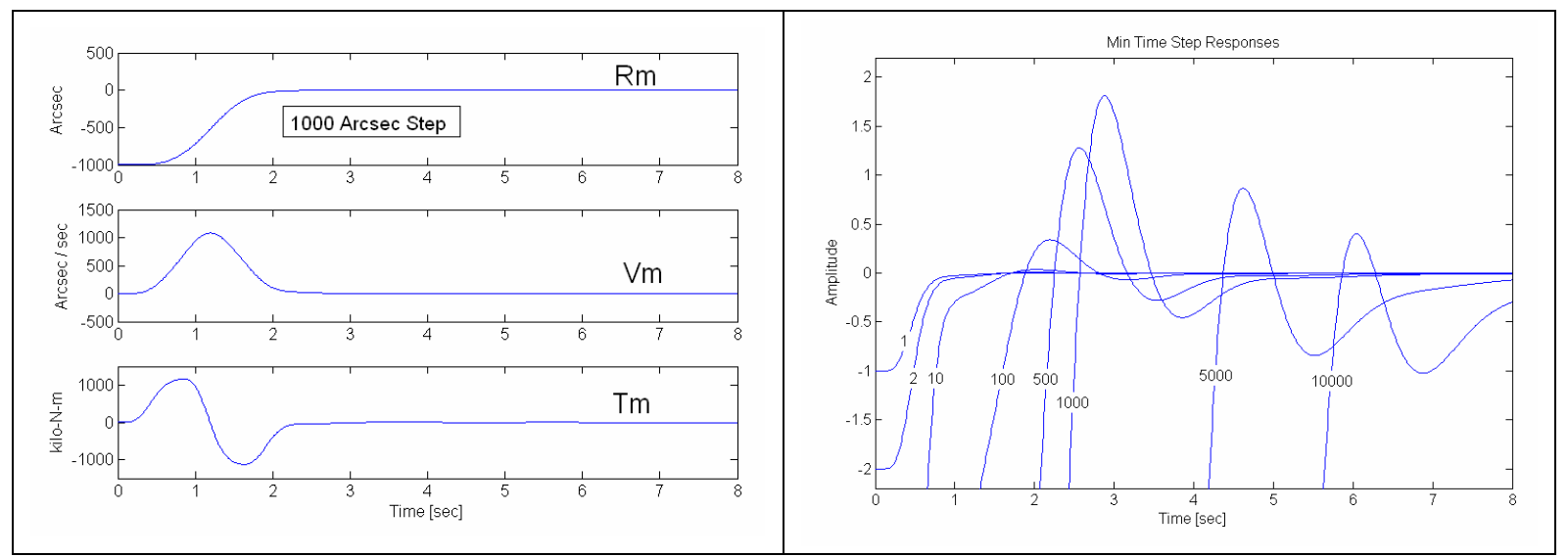

Figure 11: Offset Step Responses

\section{ACKNOWLEDGEMENT}

The authors gratefully acknowledge the support of the TMT partner institutions. They are the Association of Canadian Universities for Research in Astronomy (ACURA), the California Institute of Technology and the University of California. This work was supported as well by the Gordon and Betty Moore Foundation, the Canada Foundation for Innovation, the Ontario Ministry of Research and Innovation, the National Research Council of Canada, the Natural Sciences and Engineering Research Council of Canada, the British Columbia Knowledge Development Fund, the Association of Universities for Research in Astronomy (AURA) and the U.S. National Science Foundation.

\section{REFERENCES}

1. Szeto, K., et. al., "TMT Telescope Structure System - Design and Development Progress Report," SPIE Astronomical Telescopes and Instrumentation, Marseilles, France, $23-28$ June 2008, SPIE 7012-88.

2. Sirota, M. J., P. M. Thompson, and H. R. Jex, "Azimuth / Elevation Servo Performance of the W. M. Keck Telescope,” SPIE Symposium on Astronomical Telescopes and Instrumentation, Kona, Hawaii, 13-18 March 1994.

3. MacMynowski, D. G., Blaurock, C., and Angeli, G. Z., "Dynamic Analysis of TMT", SPIE Astronomical Telescopes and Instrumentation, Marseilles, France, 23-28 June 2008. SPIE 7017-31. 